Géométrie différentielle/Differential Geometry

\title{
Cohomologie de l'algèbre de Lie des opérateurs différentiels sur une variété, à coefficients dans les fonctions
}

\author{
Norbert PONCIN \\ Département de Mathématique, Centre Universitaire de Luxembourg, \\ Avenue de la Faïencerie, 162 A, L-1511 Luxembourg
}

Résumé. L'espace des $q$-cochaînes locales de l'algèbre de Lie des opérateurs différentiels sur une variété, à coefficients dans les fonctions, est naturellement bigradué. On ordonne totalement les termes homogènes d'une cochaîne et on représente les dérivées symboliquement par des formes linéaires sur $\mathbb{R}^{m}$. Cela conduit à une méthode permettant de calculer les trois premiers espaces de cohomologie.

Cohomology of the Lie algebra of differential operators on a manifold, with coefficients in the space of functions

Abstract. The space of local q-cochains of the Lie algebra of differential operators on a manifold, with
coefficients in the space of functions, is naturally graded. The homogeneous terms of a cochain
are totally ordered and the derivatives may be symbolized by linear forms on $\mathbb{R}^{m}$. This leads
to a method giving the first three cohomology spaces.

\section{Abridged English Version}

If $M$ is a smooth manifold and $N$ is the space of smooth functions on $M$, denote by $(A(N),[.,]$.$) the Nijenhuis-Richardson graded Lie algebra of N([2])$. The space $\mathcal{E}=A(N)_{l o c, n . c .}$ of local i.e. support preserving elements of $A(N)$, which are vanishing on $1 \in N$, is a graded Lie subalgebra. Let $H_{a l t}(\mathcal{E})_{-1 \text {, loc }}$ be the graded cohomology of $\mathcal{E}$ associated to the adjoint representation, all cochains being support preserving and of weight -1 . Observe that $\mathcal{E}^{0}=$ $g l(N)_{l o c, n . c .}$ is a Lie algebra, subalgebra of $g l(N)$. It can be shown ([1]) that the local ChevalleyEilenberg cohomology $H\left(\mathcal{E}^{0}, N\right)_{l o c}$ (of the Lie algebra $\mathcal{E}^{0}$ of differential operators on $M([3]$ ), valued in $N)$ is a part of the graded cohomology :

$$
H_{\text {alt }}(\mathcal{E})_{-1, l o c}=H(\operatorname{ker} \theta) \oplus H\left(\mathcal{E}^{0}, N\right)_{l o c} \quad,
$$

where $\theta$ is the restriction mapping of graded cochains to $\mathcal{E}^{0} \times \cdots \times \mathcal{E}^{0}$.

The purpose of this note is to give an idea of the proof of the following result :

THEOREM. - If $M$ is a smooth connected second-countable Hausdorff manifold of dimension $m \geq 3$, the space $H^{q}\left(\mathcal{E}^{0}, N\right)_{l o c}(q \in\{1,2,3\})$ is isomorphic to the corresponding space $H_{D R}^{q}(M)$ of the de Rham cohomology of $M$.

Let $E$ be a certain space constructed on an arbitrary manifold $V$ and let $U$ be an open subset of $V$. We denote by $E_{U}$ the space of same type than $E$, on $U$.

PROPOSITION. - If $H^{q}\left(\mathcal{E}_{U}^{0}, N_{U}\right)_{l o c}=0$, for each $q \in\{1, \ldots, n\} \quad\left(n \in \mathbb{N}^{*}\right)$ and each contractible domain $U$ of chart, then $H^{q}\left(\mathcal{E}^{0}, N\right)_{l o c} \approx H_{D R}^{q}(M)$, for each $q \in\{1, \ldots, n\}$. 
In the sequel, $\Omega$ is an open, possibly contractible, subset of $\mathbb{R}^{m}$. We set $\mathcal{E}^{0}=\mathcal{E}_{\Omega}^{0}$ and $N=N_{\Omega}$. Moreover, we identify the space of homogeneous polynomials of degree $r$ on $\left(\mathbb{R}^{m}\right)^{*}$, with the space $\vee^{r} \mathbb{R}^{m}$ of $r$-contravariant symmetric tensors on $\mathbb{R}^{m}$.

In view of the preceding result, it suffices to evidence the

PROPOSITION. - For each $q \in\{1,2,3\}, H^{q}\left(\mathcal{E}^{0}, N\right)_{l o c}=0$.

Let us give some details concerning the method used in the proof of this assertion.

It follows from a well known theorem of J. Peetre ([3]), that the arguments $A \in \mathcal{E}^{0}=$ $g l(N)_{l o c, n . c .}$ of our cochains are locally differential operators. If we symbolize each derivative $D_{x}^{\lambda} f=D_{x^{1}}^{\lambda^{1}} \ldots D_{x^{m}}^{\lambda^{m}} f$ of $f \in N$ by the monomial $\xi^{\lambda}=\xi_{1}^{\lambda^{1}} \ldots \xi_{m}^{\lambda^{m}}$ in the components of $\xi \in\left(\mathbb{R}^{m}\right)^{*}$, we see that the subspace $\operatorname{Diff}^{r} \subset \mathcal{E}^{0}\left(r \in \mathbb{N}^{*}\right)$ of all homogeneous differential operators of degree $r$, identifies with the space $C^{\infty}\left(\Omega, \vee^{r} \mathbb{R}^{m}\right)$. Hence, the restriction $T_{r}$ to Diff ${ }^{r}$ of any 1-cochain $T$, is a local $N$-valued operator on $C^{\infty}\left(\Omega, \vee^{r} \mathbb{R}^{m}\right)$. Applying again Peetre's theorem, shows that $T_{r}$ also is a locally differential operator, which may be symbolized by a smooth function on $\Omega$ valued in the polynomials in $\eta \in\left(\mathbb{R}^{m}\right)^{*}$ with coefficients in the dual of $\vee^{r} \mathbb{R}^{m}$. This notion of symbolic representation can be extended in a simple way to $q$-cochains $\left(q \in \mathbb{N}^{*}\right)$.

It is easily seen that the space of 1-cochains is naturally bigraded. The homogeneous part $T_{r}^{a}\left(a \in \mathbb{N}, r \in \mathbb{N}^{*}\right)$ of any 1-cochain $T$, is the sum of all terms of $T_{r}$ containing exactly $a$ derivatives. Thus, the bidegree $(0 / 1)$ corresponds to constant vector fields and (1/1) to linear vector fields. Generalizing this bigraduation to $q$-cochains $T\left(q \in \mathbb{N}^{*}\right)$, furnishes homogeneous terms $T_{\vec{r}}^{\vec{a}}=T_{r_{0} \ldots r_{q-1}}^{a_{0} \ldots a_{q-1}} \quad\left(a_{i} \in \mathbb{N}, r_{i} \in \mathbb{N}^{*}\right)$, called monomials of $T$, which may be totally ordered by the lexicographic order associated to the total order on columns, defined by

$$
\left(a_{i} / r_{i}\right)<\left(a_{j} / r_{j}\right) \Leftrightarrow a_{i}+r_{i}<a_{j}+r_{j} \text { ou }\left(a_{i}+r_{i}=a_{j}+r_{j} \text { et } r_{i}<r_{j}\right) .
$$

The method allowing us to show that the first three cohomology spaces are vanishing, is essentially based on the concepts of symbolic representation and ordered monomials. Indeed, symbolizing the equation $\partial T=0$, where $\partial$ is the Chevalley-Eilenberg coboundary operator, leads to a purely algebraic equation, much easier than the original; in the main, we use this equation to prove that an arbitrary monomial of a certain cohomologous cocycle is vanishing, if each preceding monomial fades.

\section{Introduction}

Les problèmes de déformation de l'algèbre $N=C^{\infty}(M)$ des fonctions d'une variété $M$, symplectique ou de Poisson, ont mis en lumière certaines structures algébriques et les cohomologies

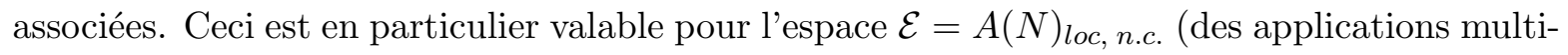
linéaires, antisymétriques de $N \times \cdots \times N$ dans $N$, qui sont locales et nulles sur les constantes), que le crochet de Nijenhuis-Richardson ([2]) munit d'une structure d'algèbre de Lie graduée, et la cohomologie graduée de $\mathcal{E}$ associée à la représentation adjointe, soit $H_{\text {alt }}(\mathcal{E})_{-1, \text { loc }}$, les indices -1 et $l o c$ indiquant qu'on se limite aux cochaînes locales, de poids -1 .

Il est clair que le terme $\mathcal{E}^{0}=A^{0}(N)_{l o c, n . c .}=g l(N)_{l o c, n . c .}$. est une algèbre de Lie, admettant $N$ comme espace de représentation canonique. On montre que la cohomologie graduée et la cohomologie $H\left(\mathcal{E}^{0}, N\right)_{l o c}$ de Chevalley locale de l'algèbre de Lie $\mathcal{E}^{0}$ des opérateurs différentiels 
sur $M([3])$, à valeurs dans les fonctions, sont liées par la relation ([1])

$$
H_{\text {alt }}(\mathcal{E})_{-1, l o c}=H(\operatorname{ker} \theta) \oplus H\left(\mathcal{E}^{0}, N\right)_{l o c},
$$

où $\theta$ désigne l'application "restriction des cochaînes alternées à $\mathcal{E}^{0} \times \cdots \times \mathcal{E}^{0}$ ".

Nous prouverons le

THEOREME 1.1. - Si $M$ est une variété de dimension $m \geq 3$, de classe $C^{\infty}$, séparée, à base dénombrable et connexe, on a $H^{q}\left(\mathcal{E}^{0}, N\right)_{l o c} \approx H_{D R}^{q}(M) \quad(q \in\{1,2,3\})$, où $H_{D R}(M)$ est la cohomologie de de Rham de $M$.

\section{Symbolisation et graduation des $q$-cochaînes}

Si $E$ représente un espace lié à une variété arbitraire $V$ et si $U$ désigne un ouvert de $V$, nous noterons $E_{U}$ l'espace de même type que $E$, construit sur $U$.

PROPOSITION 2.1. - Soit $n \in \mathbb{N}^{*}$. Si $H^{q}\left(\mathcal{E}_{U}^{0}, N_{U}\right)_{l o c}=0$, pour tout $q \in\{1, \ldots, n\}$ et pour tout domaine contractile $U$ de coordonnées locales de $M$, alors $H^{q}\left(\mathcal{E}^{0}, N\right)_{l o c} \approx H_{D R}^{q}(M)$, quel que soit $q \in\{1, \ldots, n\}$.

Dans toute la suite, $\Omega$ désigne un ouvert de $\mathbb{R}^{m}$, que nous supposerons éventuellement contractile, $\mathcal{E}^{0}=\mathcal{E}_{\Omega}^{0}$ et $N=N_{\Omega}$. D'autre part, $\vee \mathbb{R}^{m}\left(\vee^{r} \mathbb{R}^{m}\right)$ est l'espace des tenseurs symétriques ( $r$ fois) contravariants sur $\mathbb{R}^{m}$, que nous identifierons à l'espace des polynômes (homogènes de degré $r$ ) sur $\left(\mathbb{R}^{m}\right)^{*}$.

Soient $V$ et $W$ deux espaces vectoriels réels de dimension finie et $O \in g l\left(C^{\infty}(\Omega, V)\right.$, $\left.C^{\infty}(\Omega, W)\right)_{l o c}$. D'après un théorème de J. Peetre $([3])$, si $f \in C^{\infty}(\Omega)$ et $v \in V$, on a

$$
O(f v)=\sum_{\lambda} O_{\lambda}\left(\left(D^{\lambda} f\right) v\right)
$$

la série sur $\lambda=\left(\lambda^{1}, \ldots, \lambda^{m}\right) \in \mathbb{N}^{m}$ étant localement finie et les coefficients $O_{\lambda} \in C^{\infty}(\Omega, g l(V, W))$ étant univoquement déterminés par $O$ (il est clair que $D_{x}^{\lambda} f=D_{x^{1}}^{\lambda^{1}} \ldots$ $D_{x^{m}}^{\lambda^{m}} f$ ). Nous représentons les dérivées $D^{\lambda} f$ par $\eta^{\lambda}$, avec $\eta \in\left(\mathbb{R}^{m}\right)^{*}$ (où $\eta^{\lambda}=\eta_{1}^{\lambda^{1}} \ldots \eta_{m}^{\lambda^{m}}$ ), de sorte que $O$ admet le polynôme symbolisant $\varnothing \in C^{\infty}\left(\Omega, \vee \mathbb{R}^{m} \otimes g l(V, W)\right)$, défini par

$$
\varnothing(\eta ; v)=\sum_{\lambda} O_{\lambda}(v) \eta^{\lambda}
$$

Cette règle permet de symboliser les arguments $A \in \mathcal{E}^{0}=g l\left(C^{\infty}(\Omega), C^{\infty}(\Omega)\right)_{l o c, \text { n.c. de }}$ nos cochaînes, donc notamment les éléments $A_{r}$ de l'espace Diff ${ }^{r}\left(r \in \mathbb{N}^{*}\right)$ des opérateurs différentiels homogènes de degré $r$ et de constater que $\operatorname{Diff}^{r} \approx C^{\infty}\left(\Omega, \vee^{r} \mathbb{R}^{m}\right)$. Si $T$ désigne une 1-cochaîne et $T_{r}$ sa restriction à $\operatorname{Diff}^{r}$, il s'ensuit que $T_{r} \in g l\left(C^{\infty}\left(\Omega, \vee^{r} \mathbb{R}^{m}\right)\right.$, $\left.C^{\infty}(\Omega)\right)_{l o c}$, donc que

$$
T_{r}\left(f P_{r}\right)=\sum_{\lambda} T_{r, \lambda}\left(\left(D^{\lambda} f\right) P_{r}\right)
$$

$\left(f \in C^{\infty}(\Omega), \quad P_{r} \in \vee^{r} \mathbb{R}^{m}\right)$. Finalement, le polynôme symbolisant $\mathcal{T}_{r} \in C^{\infty}\left(\Omega, \vee \mathbb{R}^{m} \otimes\right.$ $\left.\left(\vee^{r} \mathbb{R}^{m}\right)^{*}\right)$ de $T_{r}$ est donné par

$$
\mathcal{T}_{r}\left(\eta ; P_{r}\right)=\sum_{\lambda} T_{r, \lambda}\left(P_{r}\right) \eta^{\lambda}
$$


L'espace des 1-cochaînes est naturellement bigradué. Le terme homogène $T_{r}^{a}$ $\left(a \in \mathbb{N}, r \in \mathbb{N}^{*}\right)$ d'une 1-cochaîne $T$ est la somme des termes de $T_{r}$ dérivant exactement $a$ fois. Il sera appelé ci-dessous monôme de $T$ de bidegré $(a / r)$. Ainsi, le bidegré $(0 / 1)$ correspond aux champs de vecteurs constants et $(1 / 1)$ aux champs linéaires.

La généralisation de cette bigraduation aux $q$-cochaînes $\left(q \in \mathbb{N}^{*}\right)$, fournit des monômes du type $T_{\vec{r}}^{\vec{a}}=T_{r_{0} \ldots r_{q-1}}^{a_{0} \ldots a_{q-1}}\left(a_{i} \in \mathbb{N}, r_{i} \in \mathbb{N}^{*}\right)$, que nous ordonnons totalement par l'ordre lexicographique associé à l'ordre total sur les colonnes, défini par

$$
\left(a_{i} / r_{i}\right)<\left(a_{j} / r_{j}\right) \Leftrightarrow a_{i}+r_{i}<a_{j}+r_{j} \text { ou }\left(a_{i}+r_{i}=a_{j}+r_{j} \text { et } r_{i}<r_{j}\right) .
$$

On étend la notion de représentation symbolique aux $q$-cochaînes $\left(q \in \mathbb{N}^{*}\right)$ et à leurs monômes.

PROPOSITION 2.2. - Il existe une correspondance biunivoque entre les monômes $T_{\vec{r}}^{\vec{a}}=$ $T_{r_{0} \ldots r_{q-1}}^{a_{0} \ldots a_{q-1}}\left(A_{0, r_{0}}, \ldots, A_{q-1, r_{q-1}}\right)$ des q-cochaînes et les applications $\mathcal{T}_{\vec{r}}^{\vec{a}}=\mathcal{T}_{r_{0} \ldots r_{q-1}}^{a_{0} \ldots a_{q-1}}\left(\eta^{0}\right.$, $\left.\ldots, \eta^{q-1} ; P_{0, r_{0}}, \ldots, P_{q-1, r_{q-1}}\right)$, qui sont de classe $C^{\infty}$ en $x \in \Omega$ et à valeurs dans l'espace $E_{\vec{r}}^{\vec{a}}$ des polynômes homogènes de degrés respectifs $a_{0}, \ldots, a_{q-1}$ en $\eta^{0}, \ldots, \eta^{q-1} \in\left(\mathbb{R}^{m}\right)^{*}$, euxmêmes à valeurs dans les formes linéaires en $P_{0, r_{0}} \in \vee^{r_{0}} \mathbb{R}^{m}, \ldots, P_{q-1, r_{q-1}} \in \vee^{r_{q-1}} \mathbb{R}^{m}$ et antisymétriques en les arguments $\left(\eta^{i}, P_{i, r_{i}}\right)$ correspondant à des colonnes égales de $(\vec{a} / \vec{r})$.

Dans la suite, nous utiliserons la même notation pour désigner un objet et sa représentation symbolique.

\section{Constance des coefficients et invariance sous $g l(m, \mathbb{R})$}

PROPOSITION 3.1. - Tout q-cocycle $\left(q \in \mathbb{N}^{*}\right)$ est, à un bord près, à coefficients constants et sans colonne (0/1) (i.e. les bidegrés de ses monômes ne contiennent pas la colonne (0/1)).

La preuve est basée sur une utilisation privilégiée de la colonne (0/1) i.e. des champs constants et une relation entre l'opérateur de cobord de Chevalley de $\mathcal{E}^{0}$ (associé à la représentation sur $N$ ) et celui de de Rham.

Le deuxième résultat escompté, l'invariance sous $g l(m, \mathbb{R})$, n'apparaît que graduellement et doit être établi séparément pour les cocycles de degré 1,2 et 3 . La principale raison de cet état des choses est liée à l'absence dans $\mathcal{E}^{0}$ d'un idéal raisonnable, supplémentaire à $g l(m, \mathbb{R})$. Notre méthode se fonde sur une utilisation privilégiée de la colonne $(1 / 1)$ i.e. des champs linéaires et une relation entre les cobords de Chevalley de $\mathcal{E}^{0}$ et de $g l(m, \mathbb{R})$ :

PROPOSITION 3.2. - Soient $T$ une q-cochaîne à coefficients constants et sans colonne $(0 / 1)$ et $T_{\vec{r}}^{\vec{a}}=T_{1 \ldots 1 r_{k_{0}} \ldots r_{q-1}}^{1 \ldots .1 a_{r_{q-1}}}\left(k_{0} \in\{0, \ldots, q\},(1 / 1)<\left(a_{k_{0}} / r_{k_{0}}\right) \leq \cdots \leq\left(a_{q-1} / r_{q-1}\right)\right)$ un monôme de T. Si d désigne le cobord de Chevalley de $\mathcal{E}^{0}$ et $\partial_{\rho}$ celui de $g l(m, \mathbb{R})$ (associé à la représentation naturelle $\rho$ sur $E_{r_{k_{0}} \ldots r_{q-1}}^{a_{k_{0} \ldots}}$ ), on a

$$
(\partial T)_{1 \vec{r}}^{1 \vec{a}}=-\partial_{\rho} T_{\vec{r}}^{\vec{a}}+\cdots,
$$

où $T_{\vec{r}}^{\vec{a}}=T_{1 \ldots 1}^{1 \ldots 1 a_{k_{0}} \ldots a_{q-1} \ldots r_{q-1}}$ est interprété comme $k_{0}$-cochaîne de $g l(m, \mathbb{R})$ à valeurs dans $E_{r_{k_{0}} \ldots r_{q-1}}^{a_{a_{0}} \ldots a_{q-1}}$ et où les termes supplémentaires, éventuels ..., sont dus à des monômes dont les bidegrés renferment au moins une colonne $(1 / 1)$ de plus que $(\vec{a} / \vec{r})$. 


\section{Preuve du théorème}

Vu 2.1., il suffit de démontrer que $H^{q}\left(\mathcal{E}^{0}, N\right)_{l o c}=0$, quel que soit $q \in\{1,2,3\}$.

Nous décrirons ici uniquement le calcul de $H^{3}\left(\mathcal{E}^{0}, N\right)_{l o c}$. Si $X \in \mathbb{R}^{m}, \eta \in\left(\mathbb{R}^{m}\right)^{*}$ et $r \in \mathbb{N}^{*}$, nous posons $X_{\eta}=\eta(X)$ et $\left(X^{r}\right)_{\eta}=\left(X_{\eta}\right)^{r}=X_{\eta}^{r}$, de façon à définir $X^{r} \in \vee^{r} \mathbb{R}^{m}$. De plus, si $X_{i} \in \mathbb{R}^{m}$ et $\eta^{j} \in\left(\mathbb{R}^{m}\right)^{*}(i, j \in\{1,2,3\})$, nous écrirons $X_{i j}$ et $T_{r \text { st }}^{a b}$ au lieu de $X_{i, \eta^{j}}$ et $T_{r s t}^{a b c}\left(\eta^{1}, \eta^{2}, \eta^{3} ; X_{1}^{r}, X_{2}^{s}, X_{3}^{t}\right)$.

L'application de (1) montre que le monôme minimum $T_{111}^{111}$ d'un 3-cocycle arbitraire $T$ est donné par $T_{111}^{111}=k \alpha\left(\operatorname{tr}_{3}\right)$, où $k \in \mathbb{R}$ et où $\alpha$ désigne l'opérateur d'antisymétrisation et $\operatorname{tr}_{3}$ la trace d'un produit de trois matrices. Or, la constante $k$ ne peut être annulée à ce stade des calculs. Il est facile de voir que les monômes $T_{11 t}^{11} c((1 / 1)<(c / t))$ sont nuls, mais $T_{111}^{111}$ engendre, lors de l'utilisation de (1) dans l'étude de certains monômes du type $T_{1 s t}^{1 b c}((1 / 1)<(b / s) \leq(c / t))$, des termes supplémentaires, qui ne sont pas en somme directe avec le bord de $g l(m, \mathbb{R})$. Ces termes constituent cependant eux-mêmes un bord de $g l(m, \mathbb{R})$ et finalement,

$$
T_{1 \text { s } t}^{1 b c}=k X_{13} X_{21} X_{32}^{t} \delta\left(\begin{array}{ccc}
1 & b & c \\
1 & s & t
\end{array}\right),\left(\begin{array}{ccc}
1 & t & 1 \\
1 & 1 & t
\end{array}\right)+\operatorname{tr} \otimes U_{s t}^{b c}
$$

où $\delta$ désigne le symbole de Kronecker et où $U_{s t}^{b c}$ appartient à l'espace $E_{s t \text {, inv }}^{b c}$ des éléments de $E_{s t}^{b c}$, qui sont invariants par $g l(m, \mathbb{R})$. Dans le cas des monômes $T_{r s t}^{a b c}((1 / 1)<(a / r) \leq(b / s) \leq$

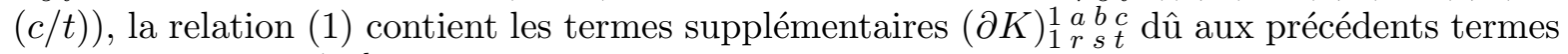
en $k$ et $(\partial(\operatorname{tr} \otimes U))_{1}^{1} \begin{aligned} & a \\ & \text { r s }\end{aligned}$ ordres considérés. Une sorte d'antilinéarité pour la trace, du cobord de Chevalley de $\mathcal{E}^{0}$, permet

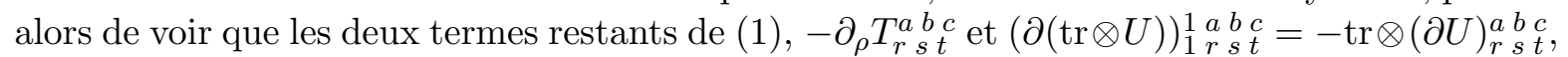
sont en somme directe. La nullité du premier implique l'invariance globale de $T$. Celle du second entraîne que $U$ est un 2-cocycle de $\mathcal{E}^{0}$, donc un 2-bord, conclusion conduisant à la suppression de la quasi-totalité des termes du type produit tensoriel dans $T_{1}^{1}$ sc $c$ :

$$
T_{1 \text { s t }}^{1 \text { bc }}=\left(k X_{13} X_{21} X_{32}^{t}+k^{\prime} X_{11} X_{23} X_{32}^{t}\right) \delta\left(\begin{array}{ccc}
1 & b & c \\
1 & s & t
\end{array}\right),\left(\begin{array}{ccc}
1 & t & 1 \\
1 & 1 & t
\end{array}\right),
$$

où $k^{\prime} \in \mathbb{R}$. Vu l'invariance de $T$, on sait à présent prouver que $k=0$, mais $k^{\prime}$ ne pourra être annulé que plus tard : un 3-cocycle arbitraire est cohomologue à un cocycle $T$ à coefficients constants, invariant et sans colonnes (0/1) et (1/1), sauf que $T_{11 t}^{1} t_{t}^{1}=k^{\prime} X_{11} X_{23} X_{32}^{t} \quad(t \geq 2)$.

L'étude des monômes à une ou plusieurs colonnes $(0 / 2)$ est essentiellement une récurrence sur le bidegré. De plus, pour annuler un monôme $T_{2}^{0} b_{\text {st }}^{c}((0 / 2)<(b / s) \leq(c / t), b+c=2+s+t)$, nous corrigeons $T$ systématiquement par les bords $\partial V$ des cochaînes invariantes, à coefficients constants, admettant les monômes minima $(\partial V)_{2}^{0} \begin{array}{ll}0 & c \\ s & \text {. }\end{array}$.

L'organigramme des cas à distinguer ne peut être donné ici. Signalons toutefois l'apparition d'un monôme critique, $T_{2 t-1 t}^{0 t+1 t}=C_{t} X_{01} X_{02} X_{12}^{t-1} X_{21}^{t} \quad\left(t \geq 3, C_{t} \in \mathbb{R}\right)$, difficile à annuler. On note alors que lors de l'étude de $T_{21}^{02 t-12} 2 t-2$, on corrige $T$ notamment par $\partial \mathcal{V}_{2 t-1,1}=$ $\partial\left(\alpha\left(\beta_{2 t-1} X_{12} X_{21}^{2 t-1}\right)\right)$ et $\partial \mathcal{V}_{2 t-1,2}=\partial\left(\alpha\left(\varepsilon_{2 t-1} X_{12}^{2} X_{21}^{2 t-2}\right)\right)\left(\beta_{2 t-1}, \varepsilon_{2 t-1} \in \mathbb{R}, \beta_{2 t-1}\right.$ arbitraire). Les monômes minima de ces bords étant $\left(\partial \mathcal{V}_{2 t-1,1}\right)_{2}^{0}{ }_{2}^{2 t-1}{ }_{2 t-2}^{2}=-2 \beta_{2 t-1} X_{01} X_{02} X_{12} X_{21}^{2 t-2}$ et $\left(\partial \mathcal{V}_{2 t-1,2}\right)_{2}^{0}{ }_{1}^{2 t-1}{ }_{2 t-2}^{2}=-2 \varepsilon_{2 t-1} X_{01} X_{02} X_{12} X_{21}^{2 t-2}$, il est possible de corriger $\partial \mathcal{V}_{2 t-1,1}$, de manière à obtenir un bord $\partial\left(\mathcal{V}_{2 t-1,1}+\mathcal{V}_{2 t-1,2}\right)$ de monôme minimum naturel, nul. Ce type de construction, réitéré, fournit un bord $\partial \mathcal{V}_{2 t-1}$, admettant le monôme minimum $\left(\partial \mathcal{V}_{2 t-1}\right)_{2 t-1 t}^{0 t+1 t}=$ $(-1)^{t+1} 2 \beta_{2 t-1} X_{01} X_{02} X_{12}^{t-1} X_{21}^{t}$. Le monôme critique $T_{2 t-1 t}^{0 t+1 t}=C_{t} X_{01} X_{02} X_{12}^{t-1} X_{21}^{t}(t \geq 3)$ peut donc être annulé, grâce au choix convenable de $\beta_{2 t-1}(2 t-1 \in\{5,7,9, \ldots\})$. 
Le cocycle étudié étant désormais sans colonne $(0 / 2)$, on voit enfin que $k^{\prime}=0$ : tout 3 -cocycle est cohomologue à un cocycle à coefficients constants, invariant et sans colonnes $(0 / 1),(1 / 1)$ et $(0 / 2)$.

Une nouvelle récurrence sur le bidegré et l'étude de certains monômes $T_{r}^{a}$ s $c$ t à partir d'un type d'équations $(\partial T)_{R S T U}^{A B C D}=0$, où $(\partial T)_{R S T}^{A B S}$ D n'est pas le plus petit monôme du bord auquel $T_{r \text { st }}^{a b c}$ contribue, permettent d'annuler tous les monômes restants.

Ici encore, la place nous manque pour décrire utilement l'organigramme des cas à distinguer. Un autre monôme critique

$$
T_{11}^{2 t-11}{ }_{t}^{1}=\left[\partial\left(\alpha\left(\frac{-2}{t(t-1)} C_{t, 1} X_{12} X_{21}^{t}\right)\right)\right]_{11 \quad t}^{2 t-11}\left(t \geq 4, C_{t, 1} \in \mathbb{R}\right)
$$

se manifeste, le monôme minimum de ce bord étant de bidegré $(0 t 2 / 21 t-1)$. Or, en considérant $T_{2}^{0 t 2} t_{t-1}$, on corrige $T$ en particulier par $\partial \mathcal{V}_{t, 1}=\partial\left(\alpha\left(\beta_{t} X_{12} X_{21}^{t}\right)\right)\left(\beta_{t}\right.$ arbitraire pour $t$ pair). On montre que le choix convenable de $\beta_{t}$, annule $C_{t, 1}$ au cours de l'étude de $T_{2}^{0 t 2}{ }_{t-1}$. Il est clair que les corrections par bords, effectuées ultérieurement, peuvent réintroduire $C_{t, 1}$, accident se produisant effectivement pour $t \in\{5,7,9, \ldots\}$. Dans ce cas, (2) peut être généralisé sous la forme

$$
T_{1 p}^{2 t-p p}{ }_{t-p+1}=\left[\partial\left(\alpha\left(\frac{(-1)^{p} 2}{t(t-1)} C_{t, 1} X_{12}^{p} X_{21}^{t-p+1}\right)\right)\right]_{1 p \quad t-p+1}^{2 t-p p}\left(p \in\left\{1, \ldots, \frac{t+1}{2}\right\}\right)
$$

où $\partial$ est à calculer formellement et $\alpha$ à supprimer, si $p=\frac{t+1}{2}$. Une équation en le dernier maillon $T_{1 \frac{t+1}{2}}^{2 \frac{t-1}{2} \frac{t+1}{2}}$ de la précédente chaîne, annule alors $C_{t, 1}$. Finalement, le monôme critique est nul, pour tout $t \geq 4: H^{3}\left(\mathcal{E}^{0}, N\right)_{l o c}=0$.

Les démonstrations détaillées de ces résultats seront présentées dans [4, 5].

Remerciements. Ce travail a été réalisé dans le cadre du projet R\&D no MEN/CUL/96/006. L'auteur remercie les Professeurs M. De Wilde et P.B.A. Lecomte pour maintes discussions fructueuses.

\section{References}

[1] Lecomte P.B.A., Melotte D. and Roger C., 1989. Explicit Form and Convergence of 1-Differential Formal Deformations of the Poisson Lie Algebra, Lett. Math. Phys., 18, p. 275-285.

[2] Nijenhuis A. and Richardson R., 1967. Deformation of Lie algebra structures, J. of Math. and Mech., 17, p. 89-105.

[3] Peetre J., 1959 et 1960. Une caractérisation des opérateurs différentiels, Math. Scand., 7 et 8, p. 211-218 et $116-120$.

[4] Poncin N., 1999. Premier et deuxième espaces de cohomologie de l'algèbre de Lie des opérateurs différentiels sur une variété, à coefficients dans les fonctions, Bull. Soc. Sc. Liège (à paraître).

[5] Poncin N., 1999. Troisième espace de cohomologie de l'algèbre de Lie des opérateurs différentiels sur une variété, à coefficients dans les fonctions, Bull. Soc. Sc. Liège (à paraître). 